\title{
Interventions to prevent hemodynamic instability during renal replacement therapy in critically ill patients: a systematic review
}

Adrianna Douvris ${ }^{1}$, Gurpreet Malhi', Swapnil Hiremath², Lauralyn Mclntyre ${ }^{3,4}$, Samuel A. Silver $^{5}$, Sean M. Bagshaw ${ }^{6}$, Ron Wald ${ }^{7}$, Claudio Ronco ${ }^{8}$, Lindsey Sikora ${ }^{9}$, Catherine Weber ${ }^{10}$ and Edward G. Clark ${ }^{2,11^{*}}$ (D)

\begin{abstract}
Background: Hemodynamic instability related to renal replacement therapy (HIRRT) may increase the risk of death and limit renal recovery. Studies in end-stage renal disease populations on maintenance hemodialysis suggest that some renal replacement therapy (RRT)-related interventions (e.g., cool dialysate) may reduce the occurrence of HIRRT, but less is known about interventions to prevent HIRRT in critically ill patients receiving RRT for acute kidney injury (AKI). We sought to evaluate the effectiveness of RRT-related interventions for reducing HIRRT in such patients across RRT modalities.

Methods: A systematic review of publications was undertaken using MEDLINE, MEDLINE in Process, EMBASE, and Cochrane's Central Registry for Randomized Controlled Trials (RCTs). Studies that assessed any intervention's effect on HIRRT (the primary outcome) in critically ill patients with AKI were included. HIRRT was variably defined according to each study's definition. Two reviewers independently screened abstracts, identified articles for inclusion, extracted data, and evaluated study quality using validated assessment tools.

Results: Five RCTs and four observational studies were included ( $n=9 ; 623$ patients in total). Studies were small, and the quality was mostly low. Interventions included dialysate sodium modeling $(n=3)$, ultrafiltration profiling $(n=2)$, blood volume $(n=2)$ and temperature control $(n=3)$, duration of RRT $(n=1)$, and slow blood flow rate at initiation $(n=1)$. Some studies applied more than one strategy simultaneously $(n=5)$. Interventions shown to reduce HIRRT from three studies (two RCTs and one observational study) included higher dialysate sodium concentration, lower dialysate temperature, variable ultrafiltration rates, or a combination of strategies. Interventions not found to have an effect included blood volume and temperature control, extended duration of intermittent RRT, and slower blood flow rates during continuous RRT initiation. How HIRRT was defined and its frequency of occurrence varied widely across studies, including those involving the same RRT modality. Pooled analysis was not possible due to study heterogeneity.

(Continued on next page)
\end{abstract}

\footnotetext{
* Correspondence: edclark@toh.ca

${ }^{2}$ Division of Nephrology, Department of Medicine and Kidney Research

Centre, Ottawa Hospital Research Institute, University of Ottawa, Ottawa, ON,

Canada

${ }^{11}$ The Ottawa Hospital - Riverside Campus, 1967 Riverside Drive, Ottawa, ON

K1H 7W9, Canada

Full list of author information is available at the end of the article
} 
(Continued from previous page)

Conclusions: Small clinical studies suggest that higher dialysate sodium, lower temperature, individualized ultrafiltration rates, or a combination of these strategies may reduce the risk of HIRRT. Overall, for all RRT modalities, there is a paucity of high-quality data regarding interventions to reduce the occurrence of HIRRT in critically ill patients.

Keywords: Acute kidney injury, Renal replacement therapy, Intradialytic hypotension, Dialysis, Hemodynamic instability

\section{Background}

Hemodynamic instability related to renal replacement therapy (HIRRT) is a frequent occurrence in critically ill patients with acute kidney injury (AKI) [1]. HIRRT complicates an estimated $30-70 \%$ of intermittent hemodialysis (IHD) treatments for AKI in the intensive care unit (ICU) [2-4]. HIRRT is also a frequent complication of other renal replacement therapy (RRT) modalities, specifically sustained low-efficiency dialysis (SLED) and continuous renal replacement therapy (CRRT) [5]. CRRT is presumed to have the least impact on the hemodynamic stability of critically ill patients [6]; nonetheless, RRT-related hypotension has still been reported to occur in 19 to $43 \%$ of patients treated with CRRT $[7,8]$.

There is evidence suggesting that HIRRT negatively impacts outcomes for patients with RRT-requiring AKI; more frequent HIRRT is associated with increased mortality [9] and may limit renal recovery after AKI [10]. Hypotensive episodes during RRT lead to decreased renal perfusion and may compromise renal recovery on that basis [10]. Accordingly, interventions to limit HIRRT across RRT modalities might ultimately improve the persistently dismal outcomes of critically ill patients with AKI treated with RRT [11-13]. As such, we sought to assess the efficacy and harms of RRT-related interventions for preventing or mitigating HIRRT in critically ill patients with AKI.

\section{Methods}

This systematic review was conducted according to a previously published protocol [14] and was registered with PROSPERO (PROSPERO 2016:CRD42016037754). A summary of the study methods follows.

\section{Study population}

We conducted a systematic review of published studies, including interventional and observational studies of critically ill adults in a medical or surgical ICU with AKI treated with RRT. Studies that involved IHD, SLED, or CRRT were included. Studies were excluded if they involved the end-stage renal disease (ESRD) population or peritoneal dialysis. Case reports, animal experiments, non-English language reports, and studies directly comparing RRT modalities were excluded.

\section{Intervention}

All included studies had a dialysis-related intervention or modifiable factor related to the application of RRT to prevent or mitigate HIRRT [14]. In a minor deviation from the previously published protocol for this systematic review [14], studies comparing dialysate buffers and filter membranes were excluded given that the use of bicarbonate-based buffers and biocompatible membranes is now standard in contemporary practice. Studies that did not include any prescribed intervention/ modifiable factor were also excluded.

\section{Comparator}

Our comparators were the groups of patients in these studies that did not receive the intervention. Observational studies without a comparator group were excluded.

\section{Outcomes}

The primary outcome was HIRRT according to the definitions provided in the individual studies. Secondary outcomes included death, ICU and hospital length of stay, renal recovery, need for interventions (vasopressor dose change, need for fluid bolus, reduced ultrafiltration goal, or cessation of ultrafiltration) to treat HIRRT, cardiovascular events, system clotting, and bleeding. We also assessed for intervention-specific harms or side effects.

\section{Study identification}

A comprehensive search strategy was developed with, and implemented by, a health information specialist (LS). Our published protocol describes the search strategy in detail [14]. An initial search of MEDLINE, PubMed, and PROSPERO yielded no prior or ongoing systematic reviews on this topic. Our search accessed the following databases: MEDLINE in Process and MEDLINE (via OVID), Embase (via OVID), and CENTRAL (via OVID). The cutoff date was 26 April 2017. To supplement our search, we also searched PubMed, reference lists, conference abstracts, and clinical trial registries. The PubMed search captured one additional publication that was missed by the initial search strategy and so the search strategy was expanded and re-run but did not yield any further articles for inclusion, including the initially missed 
article (which was still missed by the expanded strategy due to 'acute kidney injury' or 'acute renal failure' not having been used as a keyword or in the title of that particular publication).

\section{Study selection and quality assessment}

Two reviewers (AD, EGC) independently screened the study reference database for potentially eligible studies. Studies deemed potentially eligible underwent full text review. Any disagreements were resolved by consensus or discussion with a third investigator $(\mathrm{SH})$. We used the Newcastle-Ottawa Quality Assessment Scale (NOS) [15] and the Cochrane Collaboration's Tool for Assessing Risk of Bias in Randomised Trials [16] for the quality assessment of observational studies and randomized controlled trials (RCTs), respectively. For RCT quality assessment, the risk of bias was reported as low, unclear, or high risk as described by Higgins et al. [16].

\section{Data extraction and synthesis}

Two reviewers (AD, EGC) independently extracted data from all included studies. We created data extraction forms to record the following information from each study: author, year, type of study, population characteristics, intervention and comparator group, and primary and secondary outcomes. Given the small number of studies and large heterogeneity between studies, as was expected [14], we were unable to perform a meta-analysis and have presented our data as a narrative synthesis.

\section{Results}

The search process and results are depicted in Fig. 1.

A total of 840 citations were identified, of which 793 were excluded based on title and abstract. Forty-seven studies underwent full text review. Of these, 28 were excluded because there was no comparator group, five were dialysate buffer comparisons, two involved dialyzer membranes, one was unrelated to the study topic, one was a modality comparison, and one was a systematic review of bicarbonate versus lactate-buffered solutions for AKI treated with RRT [17].

In total, nine studies, consisting of five RCTs and four observational studies, met inclusion criteria and are summarized in Tables 1 and 2. Study sizes ranged from as small as 10 patients to as large as 191 patients, for a total of 623 patients. Interventions included dialysate sodium modeling $(n=3)$, ultrafiltration profiling $(n=2)$, blood volume $(n=2)$ and temperature control $(n=3)$,

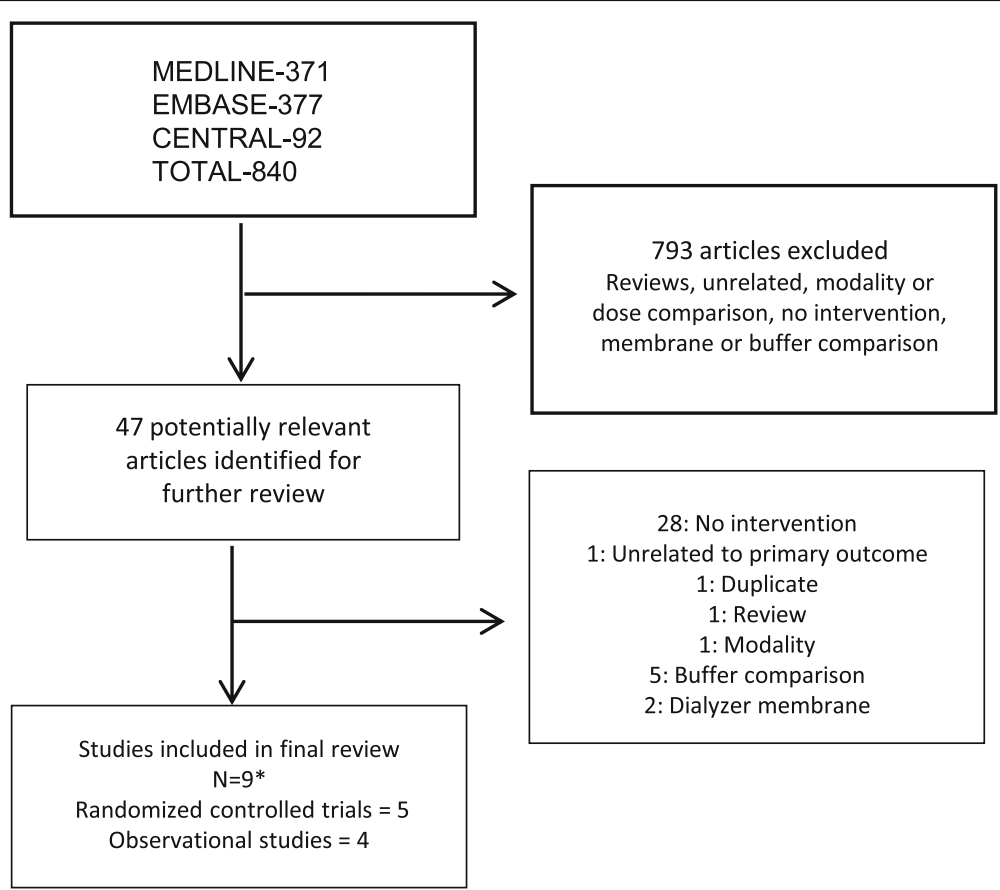

Fig. 1 Flow diagram of included studies and exclusions. Initial search of MEDLINE/PubMed and Prospero yielded no prior or ongoing systematic reviews on this topic. A health information specialist constructed and implemented the comprehensive search strategy. *Not included in the diagram: one RCT that meets inclusion criteria was not identified using this search strategy but rather, using a PubMed search, likely because the term 'acute kidney injury' or 'acute renal failure' was not in the title or listed as a keyword, and our search strategy was designed to capture studies of acute kidney injury and renal replacement therapy. Given the missed study, the search strategy was expanded, and identified 181 additional articles. Again, the same study was missed for the reason above. Five additional studies from the second search underwent full text review but were ultimately excluded because they included dose comparisons, dialysate buffer, and dialyzer membrane comparisons 
Table 1 Summary of study designs, outcomes, and definitions of HIRRT

\begin{tabular}{|c|c|c|c|c|c|c|c|c|}
\hline Study & $\begin{array}{l}\text { Setting and } \\
\text { country }\end{array}$ & Intervention & Study design & Sample size & Mean age & $\begin{array}{l}\text { Male } \\
(\%)\end{array}$ & $\begin{array}{l}\text { Primary } \\
\text { outcome(s) }\end{array}$ & HIRRT definition \\
\hline \multicolumn{9}{|c|}{ Intermittent hemodialysis } \\
\hline $\begin{array}{l}\text { Lynch (2016) } \\
\text { [21] }\end{array}$ & $\begin{array}{l}\text { USA } \\
\text { Single center } \\
\text { Medical/ } \\
\text { surgical } \\
\text { ICU }\end{array}$ & $\begin{array}{l}\text { Dialysate } \mathrm{Na}^{+} \\
\text {modeling }\end{array}$ & $\begin{array}{l}\text { Retrospective } \\
\text { cohort }\end{array}$ & $\begin{array}{l}n=191 \\
\text { RRT }=892 \\
(242 / 892 \\
\text { Na modeling) }\end{array}$ & $62 \pm 17$ & 60.7 & $\begin{array}{l}\text { In-hospital death } \\
\text { or dialysis } \\
\text { dependence at } \\
\text { discharge } \\
\text { HIRRT }\end{array}$ & $\begin{array}{l}\mathrm{SBP}<80 \mathrm{mmHg} \text {, } \\
\text { or } 50 \mathrm{mmHg} \text { drop } \\
\text { from pre-HD BP, } \\
\text { and/or start of } \\
\text { vasopressor } \\
\text { during HD }\end{array}$ \\
\hline $\begin{array}{l}\text { du Cheyron } \\
\text { (2013) [20] }\end{array}$ & $\begin{array}{l}\text { France } \\
\text { Single center } \\
\text { Medical ICU }\end{array}$ & $\begin{array}{l}\text { Blood volume } \\
\text { and temperature } \\
\text { control }\end{array}$ & $\mathrm{RCT}$ & $\begin{array}{l}n=74 \\
\text { RRT }=574\end{array}$ & $65 \pm 10$ & 68 & $\begin{array}{l}\text { HIRRT } \\
\text { Arrhythmias } \\
\text { RRT-related } \\
\text { complication }\end{array}$ & $\begin{array}{l}\mathrm{SBP}<90 \mathrm{mmHg} \\
\text { justifying } \\
\text { intervention }\end{array}$ \\
\hline $\begin{array}{l}\text { du Cheyron } \\
\text { (2010) [19] }\end{array}$ & $\begin{array}{l}\text { France } \\
\text { Single center } \\
\text { Medical ICU }\end{array}$ & $\begin{array}{l}\text { Blood volume } \\
\text { and temperature } \\
\text { control }\end{array}$ & $\begin{array}{l}\text { Prospective } \\
\text { cohort }\end{array}$ & $\begin{array}{l}n=62 \\
\text { RRT }=572\end{array}$ & $60(57-70)$ & 48.4 & $\begin{array}{l}\text { HIRRT } \\
\text { Interventions } \\
\text { Arrhythmias }\end{array}$ & $\begin{array}{l}\mathrm{SBP}<90 \mathrm{mmHg} \text { or } \\
\text { fall }>40 \mathrm{mmHg}\end{array}$ \\
\hline $\begin{array}{l}\text { Schortgen } \\
(2000)[22]\end{array}$ & $\begin{array}{l}\text { France } \\
\text { Single center } \\
\text { Medical ICU }\end{array}$ & $\begin{array}{l}\text { "Guidelines" for } \\
\text { IDH in AKI }\end{array}$ & $\begin{array}{l}\text { Retrospective } \\
\text { cohort }\end{array}$ & $\begin{array}{l}n=121 \\
\text { RRT }=537\end{array}$ & $57-60 \pm 15$ & 25.6 & $\begin{array}{l}\text { HIRRT, intervention, } \\
\text { length of stay, } \\
\text { mortality }\end{array}$ & $\begin{array}{l}\text { SBP drop }>10 \% \\
\text { from baseline or } \\
\text { infusion need }\end{array}$ \\
\hline $\begin{array}{l}\text { Paganini } \\
\text { (1996) [26] }\end{array}$ & $\begin{array}{l}\text { USA } \\
\text { Single center } \\
\text { ICU }^{*}\end{array}$ & $\begin{array}{l}\text { Variable dialysate } \\
\mathrm{Na}^{+} \text {and UF } \\
\text { modeling }\end{array}$ & $\begin{array}{l}\text { RCT with } \\
\text { crossover } \\
\text { design }\end{array}$ & $\begin{array}{l}n=10 \\
\text { RRT }=60\end{array}$ & $64.2 \pm 13.7$ & 80 & $\begin{array}{l}\text { Hemodynamics } \\
\text { Volume removal, } \\
\text { blood volume } \\
\text { change }\end{array}$ & $\begin{array}{l}\text { Interventions: } \\
\text { volume } \pm \\
\text { vasopressors }\end{array}$ \\
\hline \multicolumn{9}{|c|}{ Sustained low-efficiency dialysis } \\
\hline $\begin{array}{l}\text { Albino } \\
\text { (2014) [24] }\end{array}$ & $\begin{array}{l}\text { Brazil } \\
\text { Single center ICU*ף }\end{array}$ & $\begin{array}{l}\text { Duration of } \\
\text { dialysis: } 6 \text { vs } \\
10 \mathrm{~h}\end{array}$ & RCT & $\begin{array}{l}n=75 \\
\text { RRT }=195\end{array}$ & $61.8 \pm 15.1$ & 70.6 & $\begin{array}{l}\text { HIRRT, renal } \\
\text { recovery, mortality }\end{array}$ & $\begin{array}{l}\mathrm{SBP}<90 \mathrm{mmHg} \\
\mathrm{MAP}<60 \mathrm{mmHg}\end{array}$ \\
\hline $\begin{array}{l}\text { Lima (2012) } \\
\text { [23] }\end{array}$ & $\begin{array}{l}\text { Brazil } \\
\text { Single center } \\
\text { Medical ICU }\end{array}$ & $\begin{array}{l}\text { Lower } \\
\text { temperature, } \\
\text { dialysate } \mathrm{Na}^{+} \\
\text {and UF profiling }\end{array}$ & RCT & $\begin{array}{l}n=39 \\
\mathrm{RRT}=62\end{array}$ & $58 \pm 16$ & 67.7 & $\begin{array}{l}\text { HIRRT, length of } \\
\text { stay, mortality }\end{array}$ & $\begin{array}{l}\mathrm{SBP}<90 \mathrm{mmHg} \\
\mathrm{MAP}<60 \mathrm{mmHg} \\
\text { Interventions }\end{array}$ \\
\hline \multicolumn{9}{|c|}{ Continuous renal replacement therapy } \\
\hline $\begin{array}{l}\text { Robert } \\
\text { (2012) [25] }\end{array}$ & $\begin{array}{l}\text { France } \\
\text { Single center } \\
\text { Medical/ } \\
\text { surgical } \\
\text { ICU }\end{array}$ & Temperature & $\begin{array}{l}\text { RCT with } \\
\text { crossover } \\
\text { design }\end{array}$ & $\begin{array}{l}n=30 \\
\text { time }=12 \mathrm{~h}\end{array}$ & $66.5 \pm 10.3$ & 70 & $\begin{array}{l}\text { Hemodynamic } \\
\text { tolerance }\end{array}$ & $\begin{array}{l}\text { Fall in MAP > 20\% } \\
\text { or intervention }\end{array}$ \\
\hline $\begin{array}{l}\text { Eastwood } \\
(2012)[18]\end{array}$ & $\begin{array}{l}\text { Australia } \\
\text { Single center } \\
\mathrm{ICU}^{*}\end{array}$ & $\begin{array}{l}\text { CRRT pump } \\
\text { speed }\end{array}$ & $\begin{array}{l}\text { Prospective } \\
\text { cohort }\end{array}$ & $\begin{array}{l}n=21 \\
\text { RRT }=41 \\
\text { starts }\end{array}$ & $58+/-19.9$ & 48 & $\begin{array}{l}\text { Hemodynamic } \\
\text { parameters }\end{array}$ & $\begin{array}{l}\text { Vasopressors, fluid } \\
\text { bolus at 10, } 30 \text { min } \\
\text { Hypotension not } \\
\text { defined }\end{array}$ \\
\hline
\end{tabular}

*Type of ICU (medical, surgical, or both) not specified

"All included patients had acute kidney injury (AKI) associated with sepsis, and were on a norepinephrine infusion $(0.3-0.7 \mu \mathrm{g} / \mathrm{kg} / \mathrm{min})$

CRRT continuous renal replacement therapy, HD hemodialysis, HIRT hemodynamic instability during renal replacement therapy, MAP mean arterial pressure,

$\mathrm{Na}^{+}$sodium, RCT randomized controlled trial, RRT renal replacement therapy, SBP systolic blood pressure, SLED sustained low-efficiency dialysis, UF ultrafiltration

duration of RRT ( $n=1)$, and slow blood flow rate at initiation $(\mathrm{n}=1)$. Some studies applied more than one strategy simultaneously $(n=5)$.

Table 3 reports on the overall incidence of HIRRT across studies. Notably, no studies specifically assessed potential adverse effects (or side effects) of interventions to prevent HIRRT.

Quality assessment, using the NOS for observational studies, is reported in Table 4 and the Cochrane Collaboration risk of bias for RCTs is reported in Table 5. For the observational studies, three received at least 7/9 stars. For the RCTs, none were considered to have low risk of bias and some types of bias could not be determined based on the information provided.

Study designs, outcomes, and definitions of HIRRT used by the included studies are summarized in Table 1. There was wide variability in the definition of HIRRT used in studies both within and across the RRT modalities involved. This was particularly evident for the studies involving IHD, where each used a different definition. There was a consistent definition used by the two studies employing SLED, defining HIRRT as a systolic blood pressure $(\mathrm{SBP})<90 \mathrm{mmHg}$ or mean arterial pressure (MAP) $<60 \mathrm{mmHg}$. HIRRT definitions were different for the two studies employing CRRT; Eastwood et al. [18] used a 
Table 2 Comparison of renal replacement therapy prescriptions, achieved ultrafiltration goals, and duration of treatment

\begin{tabular}{|c|c|c|c|c|c|c|c|c|}
\hline Study & $\mathrm{Q}_{B}(\mathrm{~mL} / \mathrm{min})$ & $\begin{array}{l}\text { Temperature } \\
\left({ }^{\circ} \mathrm{C}\right)^{*}\end{array}$ & $\begin{array}{l}\text { UF rate } \\
(\mathrm{mL} / \mathrm{h})\end{array}$ & UF goal (L) & $\begin{array}{l}\text { UF achieved } \\
\text { (L) }\end{array}$ & $\begin{array}{l}\text { Dialysate } \mathrm{Na}^{+} \\
(\mathrm{mmol} / \mathrm{L})\end{array}$ & $\begin{array}{l}\text { Dialysate } \\
\mathrm{Ca}^{+}(\mathrm{mmol} / \mathrm{L})\end{array}$ & Time $(h)^{*}$ \\
\hline \multicolumn{9}{|c|}{ Intermittent hemodialysis } \\
\hline $\begin{array}{l}\text { Lynch (2016) } \\
\text { [21] }\end{array}$ & $\begin{array}{l}\text { Case: } 310 \\
\text { Control: } 292\end{array}$ & $\begin{array}{l}\text { "Cooled } \\
\text { dialysate" (\%) } \\
\text { Case: } 12 \\
\text { Control: } 2.3\end{array}$ & Not specified & Median: 2.25 & $\begin{array}{l}\text { Mean } 2 \mathrm{~L} \\
\text { No difference; } \\
38 \% \text { of sessions } \\
\text { did not reach goal }\end{array}$ & $\begin{array}{l}\text { Modeling: } \\
\text { not specified } \\
\text { Fixed: } 140\end{array}$ & 1.25 & $\begin{array}{l}\text { Case: } 3.37 \\
\text { Control: } 3\end{array}$ \\
\hline $\begin{array}{l}\text { du Cheyron } \\
\text { (2013) [20] }\end{array}$ & $200-250$ & $\begin{array}{l}1{ }^{\circ} \mathrm{C} \text { below } \\
\text { body } \\
\text { temperature }\end{array}$ & $\begin{array}{l}\text { BVM: } 500 \\
\text { BVM + BTM: } \\
522 \\
\text { Control: } 500\end{array}$ & Not specified & $\begin{array}{l}\text { BVM: } 3.0 \\
\text { BVM + BTM: } 3.0 \\
\text { Control: } 3.0\end{array}$ & 145 & 1.75 & $>4$ \\
\hline $\begin{array}{l}\text { du Cheyron } \\
\text { (2010) [19] }\end{array}$ & $200-250$ & 36.0 & $\begin{array}{l}\text { Case: } 548 \pm 92 \\
\text { Control: } \\
415 \pm 112\end{array}$ & Not specified & $\begin{array}{l}\text { Case: } 3.0 \pm 0.64 \\
\text { Control: } 2.1 \pm 0.62\end{array}$ & 145 & 1.75 & $>4$ \\
\hline $\begin{array}{l}\text { Schortgen } \\
\text { (2000) [22] }\end{array}$ & $150-200$ & $\begin{array}{l}\text { "Guidelines": } \\
\leq 37.0 \\
\text { Control: } \\
\geq 37.0\end{array}$ & $\begin{array}{l}\text { Not specified."Sequential } \\
\text { UF" in } 15 \% \text { of cases }\end{array}$ & Not specified & $\begin{array}{l}\text { "Guidelines": } \\
-11 \pm 515 \mathrm{~mL} \\
\text { Control: }+135 \pm \\
434 \mathrm{~mL}\end{array}$ & $\begin{array}{l}\text { "Guidelines": } \\
\text { > } 145 \text { in } 67 \% \\
\text { Control: < } 145\end{array}$ & 1.75 & $\begin{array}{l}\text { "Guidelines": } \\
5.0 \pm 1.5 \\
\text { Control: } \\
4.2 \pm 1.0\end{array}$ \\
\hline $\begin{array}{l}\text { Paganini } \\
\text { (1996) [26] }\end{array}$ & 300 & Unknown & $\begin{array}{l}\text { Variable (Case) vs } \\
\text { Fixed (Control) }\end{array}$ & Not specified & $\begin{array}{l}\text { Case: } 2.0 \pm 1.2 \mathrm{~L} \\
\text { Control: } \\
1.56 \pm 1.3 \mathrm{~L}\end{array}$ & $\begin{array}{l}\text { Case: } 160 \text { to } \\
140 \\
\text { Fixed: } 140\end{array}$ & Unknown & $>4$ \\
\hline \multicolumn{9}{|c|}{ Sustained low-efficiency dialysis } \\
\hline $\begin{array}{l}\text { Albino } \\
\text { (2014) [24] }\end{array}$ & 200 & 35.5 & $\begin{array}{l}\text { Case: } 221-237 \\
\text { Control: } 288-357\end{array}$ & $\begin{array}{l}\text { Case: } 2.52- \\
2.76 \text { Control: } 1.95- \\
2.26\end{array}$ & $\begin{array}{l}\text { Case: } 2.21-2.37 \\
\text { Control: } \\
1.73-2.14\end{array}$ & $\begin{array}{l}\text { Range } 142- \\
148\end{array}$ & Unknown & $\begin{array}{l}\text { Case: } 10 \\
\text { Control: } 6\end{array}$ \\
\hline $\begin{array}{l}\text { Lima (2012) } \\
{[23]}\end{array}$ & $150-200$ & $\begin{array}{l}\text { Case: } 35.5 \\
\text { Control: } 37.0\end{array}$ & $\begin{array}{l}\text { Case: Variable } \\
\text { Control: Fixed }\end{array}$ & Not specified & $\begin{array}{l}\text { Case: } 2.23 \pm 1.2 \\
\text { Control: } \\
1.59 \pm 1.0\end{array}$ & $\begin{array}{l}\text { Case: } 150 \text { to } \\
138 \\
\text { Control: } 138\end{array}$ & 1.75 & $>6$ \\
\hline \multicolumn{9}{|c|}{ Continuous renal replacement therapy } \\
\hline $\begin{array}{l}\text { Robert } \\
\text { (2012) [25] }\end{array}$ & $150-200$ & $\begin{array}{l}\text { Heating device } \\
\text { at } 36.0 \text { or } 38.0 \\
\text { then crossover } \\
\text { at } 6 \mathrm{~h}\end{array}$ & $35 \mathrm{~mL} / \mathrm{kg} / \mathrm{h}$ & Not specified & Not specified & Not specified & Not specified & $\begin{array}{l}\text { Not specified } \\
\text { (Time period } \\
\text { for outcomes } \\
\text { assessment: } \\
\text { first } 12 \mathrm{~h} \\
\text { after initiation) }\end{array}$ \\
\hline $\begin{array}{l}\text { Eastwood } \\
\text { (2012) [18] }\end{array}$ & $\begin{array}{l}\text { Routine: increase } \\
\text { of } 50 \mathrm{~mL} / \mathrm{min} \\
\text { over } 1-4 \mathrm{~min} \\
\text { until } 200 \mathrm{~mL} / \mathrm{min} \\
\text { Slow: increase } \\
\text { of } 20-50 \mathrm{~mL} / \mathrm{min} \\
\text { over } 3-10 \mathrm{~min} \\
\text { until } 200 \mathrm{~mL} / \mathrm{min}\end{array}$ & Not specified & Not specified & Not specified & Not specified & Not specified & Not specified & $\begin{array}{l}\text { Not specified } \\
\text { (Time period } \\
\text { for outcomes } \\
\text { assessment: } \\
\text { first } 30 \text { min } \\
\text { after initiation) }\end{array}$ \\
\hline
\end{tabular}

*Unless otherwise specified

The term 'case' is used to refer to the group that received an intervention to limit hemodynamic instability related to renal replacement therapy (HIRRT), irrespective of study design

BTM blood temperature online monitoring, BVM blood volume online monitoring, Ca calcium, Na sodium, $Q_{B}$ blood flow rate, UF ultrafiltration

definition that included only assessing the need for vasopressors or fluid bolus during RRT initiation.

Table 2 also summarizes the different RRT prescriptions, achieved ultrafiltration (UF) goals, and durations of treatment across studies.

As reported in Table 3, there was wide variability in the incidence of HIRRT reported by studies within and across different RRT modalities. Detailed results are reported according to RRT modality in Additional file 1. For the four of five IHD studies that reported it, the overall occurrence of HIRRT per session ranged from $10.6 \%$ to $65.6 \%$ [19-22]. For SLED studies $(n=2)$, the overall occurrence of HIRRT per session was $38.7 \%$ [23] and 59.5\% [24]. For CRRT studies $(n=2)$, the overall occurrence of HIRRT was not reported on a sessional basis. One CRRT study reported that up to $50 \%$ of patients required interventions to treat HIRRT early after initiation of therapy [25] and the other study reported no HIRRT [18]. Study interventions shown to be effective at reducing HIRRT in IHD included: sodium and ultrafiltration profiling (in a study of only 10 patients) [26]; implementation of "guidelines" to limit HIRRT (see Additional file 1 for complete details) [22]; and online blood volume and temperature control by a small observational study [19]. However, online blood volume and temperature control was not found to be effective for IHD patients by a subsequent, larger RCT by the same group [20]. One SLED RCT ( $n=39$ patients) found that 
Table 3 Study features and overall incidence of HIRRT

\begin{tabular}{|c|c|c|c|c|c|}
\hline Study & HIRRT definition & Intervention & $\begin{array}{l}\text { Severity of illness } \\
\text { scores }\end{array}$ & $\begin{array}{l}\text { Pre-dialysis BP } \\
(\mathrm{mmHg})^{*}\end{array}$ & HIRRT \\
\hline \multicolumn{6}{|l|}{ Intermittent hemodialysis } \\
\hline Lynch (2016) [21] & $\begin{array}{l}\mathrm{SBP}<80 \mathrm{mmHg} \text {, } \\
\text { or } 50 \mathrm{mmHg} \text { drop } \\
\text { from pre-HD BP, } \\
\text { and/or start of } \\
\text { vasopressor } \\
\text { during HD }\end{array}$ & $\begin{array}{l}\text { Dialysate } \\
\text { sodium } \\
\text { modeling }\end{array}$ & $\begin{array}{l}\text { SOFA: } \\
\text { Case: } 13.0 \pm 2.0 \\
\text { Control: } \\
13.0 \pm 3.0\end{array}$ & $\begin{array}{l}\text { Case: } 119.0 \pm 16.0 \\
\text { Control: } 129.0 \pm 21.0\end{array}$ & $\begin{array}{l}\text { Case: } 36 / 242=14.9 \% \\
\text { Control: } 59 / 650=9.1 \% \\
\text { Overall: } 95 / 892=10.6 \%\end{array}$ \\
\hline du Cheyron (2013) [20] & $\begin{array}{l}\mathrm{SBP}<90 \mathrm{mmHg} \\
\text { justifying Intervention }\end{array}$ & BVM and BTM & $\begin{array}{l}\text { SOFA: } \\
\text { BVM: } 7 \text { (5-9) } \\
\text { BVM + BTM: } \\
8 \text { (4-11) } \\
\text { Control: } 8(5-10) \\
\text { Overall: } 10(8-12)\end{array}$ & $\begin{array}{l}\text { Not reported for } \\
\text { start of sessions } \\
\text { but "did not differ } \\
\text { among treatment } \\
\text { modalities at } \\
\text { any time" }\end{array}$ & $\begin{array}{l}\text { BVM: } 33 / 190=17.4 \% \\
\text { BVM + BTM: } 30 / 194=15.5 \% \\
\text { Control: } 32 / 188=17.0 \% \\
\text { Overall: } 95 / 572=16.6 \%\end{array}$ \\
\hline du Cheyron (2010) [19] & $\begin{array}{l}\mathrm{SBP}<90 \mathrm{mmHg} \\
\text { or fall }>40 \mathrm{mmHg}\end{array}$ & $\begin{array}{l}\text { Blood volume } \\
\text { and Temp } \\
\text { control }\end{array}$ & $\begin{array}{l}\text { SOFA: } \\
\text { Case: } 8.5(6-16) \\
\text { Control: } \\
8.0(5-14)\end{array}$ & Not reported & $\begin{array}{l}\text { Case: } 41 / 189=21.7 \% \\
\text { Control: } 110 / 383=28.7 \% \\
\text { Overall: } 151 / 572=26.4\end{array}$ \\
\hline Schortgen (2000) [22] & $\begin{array}{l}\text { SBP drop }>10 \% \\
\text { from baseline or } \\
\text { volume or vasopressors }\end{array}$ & $\begin{array}{l}\text { "Guidelines" } \\
\text { for HIRRT in } \\
\text { AKI }\end{array}$ & $\begin{array}{l}\text { SAPS II: } \\
\text { "Guidelines": } \\
59.0 \pm 24.0 \\
\text { Control: } \\
50.0 \pm 17.0\end{array}$ & $\begin{array}{l}\text { "Guidelines": } \\
121.0 \pm 23.0 \\
\text { Control: } \\
125.0 \pm 24.0\end{array}$ & $\begin{array}{l}\text { "Guidelines": } 176 / 289=60.9 \% \\
\text { Control: } 176 / 248=71.0 \% \\
\text { Overall: } 352 / 537=65.5 \%\end{array}$ \\
\hline Paganini (1996) [26] & $\begin{array}{l}\text { Case: volume } \pm \\
\text { vasopressors }\end{array}$ & $\begin{array}{l}\text { Variable } \\
\text { dialysate } \\
\text { sodium and } \\
\text { UF modeling }\end{array}$ & $\begin{array}{l}\text { APACHE II: } \\
\text { Overall: } \\
28.7 \pm 4.7\end{array}$ & $\begin{array}{l}\text { MAP: } \\
\text { Case: } 82.8 \pm 16.9 \\
\text { Control: } 86.2 \pm \\
18.9\end{array}$ & $\begin{array}{l}\text { Case: } 16.0 \%^{\S} \\
\text { Control: } 45.4 \%^{\S}\end{array}$ \\
\hline \multicolumn{6}{|l|}{ Slow low-efficiency dialysis } \\
\hline Albino (2014) [24] & $\begin{array}{l}\mathrm{SBP}<90 \mathrm{mmHg} \\
\mathrm{MAP}<60 \mathrm{mmHg}\end{array}$ & $\begin{array}{l}\text { Duration of } \\
\text { dialysis } 6 \text { vs } \\
10 \mathrm{~h}\end{array}$ & $\begin{array}{l}\text { SOFA: } \\
6 \text { h: } 13.1 \pm 2.4 \\
10 \text { h: } 14.2 \pm 3.0 \\
\text { Overall: } \\
13.6 \pm 2.7\end{array}$ & Not reported & $\begin{array}{l}6 \text { h: } 63 / 100=63.0 \% \\
10 \text { h: } 53 / 95=55.8 \% \\
\text { Overall: } 116 / 195=59.5 \%\end{array}$ \\
\hline Lima (2012) [23] & $\begin{array}{l}\mathrm{SBP}<90 \mathrm{mmHg} \\
\text { MAP }<60 \mathrm{mmHg} \\
\text { Interventions }\end{array}$ & $\begin{array}{l}\text { Lower } \\
\text { temperature, } \\
\text { dialysate } \\
\text { sodium and } \\
\text { UF profiling }\end{array}$ & $\begin{array}{l}\text { SOFA: } \\
\text { Case: } 12.0 \pm 3.9 \\
\text { Control: } \\
11.0 \pm 4.4\end{array}$ & $\begin{array}{l}\text { Case: } 132.0 \pm 25.0 \\
\text { Control: } 124.0 \pm \\
24.0\end{array}$ & $\begin{array}{l}\text { Case: } 8 / 34=23.5 \% \\
\text { Control: } 16 / 28=57.1 \% \\
\text { Overall: } 24 / 62=38.7 \%\end{array}$ \\
\hline \multicolumn{6}{|c|}{ Continuous renal replacement therapy } \\
\hline Robert (2012) [25] & $\begin{array}{l}\text { Therapeutic } \\
\text { intervention for } \\
\text { hypotension }\end{array}$ & $\begin{array}{l}\text { Temperature } \\
\text { setting:: } \\
\text { A: } 38^{\circ} \mathrm{C} \\
\text { then } 36^{\circ} \mathrm{C} \\
\text { B: } 36^{\circ} \mathrm{C} \\
\text { then } 38^{\circ} \mathrm{C}\end{array}$ & $\begin{array}{l}\text { SOFA: } \\
\text { A:12.8 } \pm 3.8 \\
\text { B: } 8.0 \pm 3.8 \\
\text { Overall: } \\
10.6 \pm 4.6\end{array}$ & $\begin{array}{l}\text { A: } 118.0 \pm 26.0 \\
\text { B: } 113.0 \pm 26.0 \\
\text { Overall: } 117 \pm 30\end{array}$ & $\begin{array}{l}\text { Patients requiring } \\
\text { intervention for HIRRT:\# } \\
\text { Period 1: } \\
\text { A: } 8 / 16=50.0 \% \\
\text { B: } 5 / 14=35.7 \% \\
\text { Period 2: } \\
\text { A: } 3 / 11=27.3 \% \\
\text { B:4/11 }=63.6 \%\end{array}$ \\
\hline Eastwood (2012) [18] & $\begin{array}{l}\text { Vasopressor use } \\
\text { and/or fluid bolus } \\
\text { at } 10 \text { and } 30 \mathrm{~min}\end{array}$ & $\begin{array}{l}\text { CRRT pump } \\
\text { speed }\end{array}$ & $\begin{array}{l}\text { APACHE II: } \\
\text { Case: } 23.1 \pm 4.5 \\
\text { Control: } \\
25.9 \pm 6.6 \\
\text { Overall: } \\
24.5 \pm 5.8\end{array}$ & $\begin{array}{l}\text { MAP: } \\
\text { Case: } 82.5 \pm 15.0 \\
\text { Control: } 82.4 \pm \\
15.1 \\
\text { Overall: } 82.4 \pm \\
15.0\end{array}$ & No HIRRT reported \\
\hline
\end{tabular}

*Systolic blood pressure, unless otherwise specified

"Incidence per session (rather than per patient), unless otherwise specified

${ }^{s}$ Exact number of HIRRT events/intermittent hemodialysis sessions per group was not reported

\#Cross-over after $6 \mathrm{~h}$ (period 1 is first $6 \mathrm{~h}$; period 2 is second $6 \mathrm{~h}$ )

The term 'case' is used to refer to a group that received an intervention to limit HIRRT, irrespective of study design

$A K I$ acute kidney injury, APACHE Acute Physiology and Chronic Health Evaluation, BP blood pressure, BTM blood temperature online monitoring, BVM blood volume online monitoring, $C R R T$ continuous renal replacement therapy, HD hemodialysis; HIRRT hemodynamic instability during renal replacement therapy, MAP mean arterial pressure, SAPS Simplified Acute Physiology Score, SBP systolic blood pressure, SOFA Sequential Organ Failure Assessment, UF ultrafiltration 
Table 4 Newcastle Ottawa Scale (NOS) for quality assessment of nonrandomized studies

\begin{tabular}{|c|c|c|c|c|c|c|c|c|c|c|c|}
\hline \multirow[t]{2}{*}{ Study } & \multirow[t]{2}{*}{ Study design } & \multicolumn{4}{|c|}{ Selection } & \multicolumn{2}{|c|}{ Comparability } & \multicolumn{3}{|c|}{ Outcome } & \multirow{2}{*}{$\begin{array}{l}\text { Total } \\
\text { point }\end{array}$} \\
\hline & & $\overline{S 1}$ & $\mathrm{~S} 2$ & S3 & $\overline{54}$ & $\overline{\mathrm{C} 1}$ & $\mathrm{C} 2$ & $\overline{\mathrm{O} 1}$ & $\mathrm{O} 2$ & $\overline{\mathrm{O} 3}$ & \\
\hline Lynch (2016) [21] & Retrospective cohort & 1 & 1 & 1 & 1 & 1 & 1 & 1 & 1 & 1 & 9 \\
\hline du Cheyron (2010) [19] & Prospective cohort & 1 & 0 & 1 & 1 & 1 & 1 & 1 & 1 & 1 & 8 \\
\hline Schortgen (2000) [22] & Retrospective cohort & 1 & 0 & 1 & 0 & 0 & 1 & 1 & 1 & 1 & 6 \\
\hline Eastwood (2012) [18] & Prospective cohort & 1 & 1 & 1 & 1 & 0 & 1 & $0^{*}$ & 1 & 1 & 7 \\
\hline
\end{tabular}

*Unclear if blinded assessment

For quality assessment, $>7$ points is considered 'good quality'

lower dialysate temperature in addition to sodium and UF profiling led to less HIRRT [23]. Another SLED RCT did not find that extending SLED duration from 6 to 10 $\mathrm{h}$ led to less HIRRT [24]. A crossover RCT $(n=30$ patients) found that lower temperature at the initiation of CRRT led to improved hemodynamic stability [25], whereas a prospective cohort study ( $n=21$ patients) found no effect in slowing CRRT pump speed at the initiation of treatment [18].

\section{Discussion}

Our systematic review suggests that there is limited evidence with respect to any particular intervention's efficacy (or lack thereof) in mitigating HIRRT in critically ill patients across RRT modalities. Nonetheless, small studies indicate that the combination of higher dialysate sodium, variable UF rate, and lower temperature might reduce the incidence of HIRRT in critically ill patients with AKI.

Sodium modeling [27] may mitigate intradialytic hypotension (IDH) (a form of HIRRT) in chronic IHD. This is a strategy whereby a dialysis session begins with a high sodium dialysate concentration, which is then reduced in a step-wise manner. Improved hemodynamic tolerance with higher dialysate sodium is believed to be mediated by reducing osmotic fluid shifts between

Table 5 Quality assessment of randomized controlled trials using Cochrane Collaboration's Tool for Assessing Risk of Bias in Randomized Trials

\begin{tabular}{|c|c|c|c|c|c|c|}
\hline \multirow[t]{2}{*}{ Study } & \multicolumn{2}{|c|}{ Selection } & \multirow[t]{2}{*}{ Performance } & \multirow[t]{2}{*}{ Detection } & \multirow[t]{2}{*}{ Attrition } & \multirow[t]{2}{*}{ Reporting } \\
\hline & $\mathrm{R}$ & A & & & & \\
\hline $\begin{array}{l}\text { Albino (2014) } \\
\text { [24] }\end{array}$ & $?$ & - & + & $?$ & - & - \\
\hline $\begin{array}{l}\text { du Cheyron } \\
\text { (2013) [20] }\end{array}$ & - & - & + & $?$ & + & - \\
\hline $\begin{array}{l}\text { Lima (2012) } \\
{[23]}\end{array}$ & - & $?$ & + & $?$ & - & - \\
\hline $\begin{array}{l}\text { Robert (2012) } \\
\text { [25] }\end{array}$ & - & $?$ & + & $?$ & + & - \\
\hline $\begin{array}{l}\text { Paganini } \\
\text { (1996) [26] }\end{array}$ & $?$ & $?$ & + & $?$ & - & $?$ \\
\hline
\end{tabular}

+ High risk of bias, - low risk of bias, ? unknown risk of bias (moderate is not an option); $A$ allocation concealment, $R$ random sequence generation intravascular and interstitial compartments [28]. This can be combined with UF profiling where the UF rate is highest with higher dialysate sodium to maximize fluid removal and is reduced along with dialysate sodium concentration. The study by Lynch et al. [21], using sodium modeling in IHD, was unable to show a significant reduction in HIRRT. Nonetheless, this was a retrospective study where sodium modeling was prescribed by treating clinicians in only $27 \%$ of sessions, likely contributing to baseline differences in co-morbidities between the two groups including higher pre-IHD vasopressor requirements in the sodium modeling group. On the other hand, both RCTs that assessed combined sodium and UF profiling did find less HIRRT within the intervention group $[23,26]$. In the latter study, which also included cool dialysate in the intervention group, the sample size was small, and the control group had a significantly lower MAP pre- and post-dialysis. As such, based on the available evidence, it remains unclear if sodium profiling alone is a useful technique for limiting HIRRT in the context of AKI and critical illness, but it may be effective in combination with other strategies including UF modeling and cool dialysate.

Possible adverse effects of high dialysate sodium and sodium profiling are reported in the ESRD population on chronic IHD, and include increased thirst, interdialytic weight gain, and hypertension [29-31] which can contribute to left ventricular hypertrophy, cardiovascular events, and increased mortality [29, 32, 33]. A recent systematic review of 23 studies in the chronic IHD population found that higher dialysate sodium led to increased interdialytic weight gains but did not confirm an association with an increased risk of death [34]. The authors concluded that further research is needed to assess the impact of dialysate sodium on mortality [34]. Our included studies reported that post-session sodium levels were similar between groups but did not provide data on adverse effects or fluid balance. This is particularly relevant given the mounting evidence of a strong association between fluid overload and increased mortality in the AKI population [35-38].

There is increasing evidence to support the use of cooled dialysate to limit IDH in outpatient IHD patients 
[39-42]. In addition, two systematic reviews of cool dialysate in the chronic IHD population did not identify any trials that included an assessment of adverse effects such as mortality, cardiovascular events, access failure, or bleeding [43]. Cooler dialysate promotes vasoconstriction by reducing heat transfer from the dialysate and may mitigate myocardial stunning [39], a phenomenon that has also recently been shown to occur in patients with AKI being treated with IHD [44] and CRRT [45]. The small pilot study by Robert et al. [25] found that decreasing the fluid warmer temperature from $38{ }^{\circ} \mathrm{C}$ to $36{ }^{\circ} \mathrm{C}$ at the start of CRRT improved hemodynamics but did not impact body temperature. This study did not comment on adverse effects related to hypothermia, but mean body temperature did not fall below $36{ }^{\circ} \mathrm{C}$. Studies involving CRRT have shown that mild decreases in core body temperature result in increased systematic vascular resistance and decreasing oxygen consumption [46, 47]. However, prolonged and extreme hypothermia in the broader ICU population may correlate with organ dysfunction and increased ICU mortality [48]. A study examining the effect of cooling on critically ill febrile patients suggested that hypothermia induced by CRRT results in immune system dysfunction [49] and that an assessment of longer term outcomes in this population is particularly warranted.

Blood volume monitoring has been utilized in the chronic IHD population as a means to predict and thereby prevent hemodynamic instability during treatment [50,51]. Two prospective observational studies of relative blood volume monitoring in the AKI population on IHD did not find any significant concordance between blood volume monitoring and hypotension $[2,4]$. We identified two studies by du Cheyron et al. (2010 and 2013), an observational study [19] followed by an RCT [20], the latter of which found no significant impact on HIRRT or other dialysis-related complications. This suggests that online blood volume monitoring may not have any benefit beyond that which might be provided by cooled dialysate, high dialysate sodium and calcium concentration, and variable UF rate, all of which were part of the standard dialysis prescription. There also may be physiological differences between central and peripheral blood volume, and plasma refilling from dialysis fluid shifts is thought to occur primarily from peripheral rather than central compartments [52]. Consequently, this process may not be reflected in blood volume monitoring from central venous catheters. Interestingly, a recent small study found that low baseline peripheral perfusion index (PPI) measured by pulse oximetry could predict hypotension during continuous venovenous hemofiltration $(\mathrm{CVVH})$ in the ICU [53].

Patients at higher risk of complications, given hemodynamic instability at baseline, are more likely to be selected for treatment with CRRT (or SLED). Hypotension at CRRT initiation has been reported in $18.8-25.0 \%$ of patients [8, 54]. Kim et al. [54] also assessed hypotension in relation to CRRT initiation and found that it affected $7.8 \%$ of circuit starts. Eastwood et al. [18] compared CRRT routine and slow blood flow rates at initiation and reported no hypotensive events in either arm. However, the study population from Kim et al. as compared to Eastwood et al. was, on average, 10 years older $(65.9 \pm 11.5$ years vs $58 \pm 19.9$ years $)$ and had a lower MAP at baseline $(69.9 \pm 9.9 \mathrm{mmHg}$ vs $84.2 \pm 15.0$ $\mathrm{mmHg}$ ( $p$ value not provided).

Our systematic review suggests that HIRRT is a common phenomenon across RRT modalities utilized for the treatment of AKI, complicating approximately 10-70\% of IHD sessions [19-22, 26], approximately $40-60 \%$ of SLED sessions [23, 24], and up to $50 \%$ of CRRT sessions [25]. Part of the variability in the frequency of HIRRT observed across studies is most likely attributable to variations in the definition of HIRRT being used, as well as other aspects of how and when different RRT modalities are applied. In comparison, the outpatient IHD definition for IDH has three components: 1) a drop in SBP of $20 \mathrm{mmHg}$ or drop in MAP of at least $10 \mathrm{mmHg}$; 2) presence of symptoms of end organ ischemia; and 3) intervention carried out by dialysis staff [55]. However, this definition of IDH cannot be readily applied to ICU patients as many are receiving concurrent vasopressor and/or inotropic support, and it is often not possible to assess for ischemic symptoms. This highlights the importance of better defining HIRRT in the context of critical illness as a focus for future research.

The data presented in this systematic review must be interpreted in the context of its limitations. There was substantial heterogeneity among the included studies because of multiple RRT-related interventions, different RRT modalities, and variability in the definition of HIRRT as discussed. Another important limitation is that included studies, and hence our review, did not assess the potential for adverse effects of interventions to limit HIRRT. Also, the timing of the onset of HIRRT within a session was not provided, with the exception of Schortgen et al. [22], and Eastwood et al. [18]. Whether HIRRT occurs at RRT initiation or later during the session has physiological relevance, as one would not expect fluid removal to be the main culprit at session onset. However, rapid fluid shifts between compartments, myocardial stunning, or peripheral vasodilation could precipitate HIRRT early on. With regards to study quality, many RCTs had small sample sizes, and the majority were unblinded. Retrospective studies had important baseline differences between cohorts as interventions were likely prescribed for a clinical reason. The total number of patients from all included studies was only 
623 and our systematic review is very likely to have been underpowered to assess most outcomes. This also highlights the extent to which this area is ripe for further study. While this review focused on RRT-related interventions and HIRRT, the impact of different RRT modalities on HIRRT and other outcomes is, unto itself, a controversial aspect of RRT administration in critically ill patients [6]. Nonetheless, the impact of RRT modality on mortality and renal recovery has been the subject of prior reviews [56-60] and was considered beyond the scope of this one.

There are also notable strengths to this study. The search strategy was comprehensive and was conducted according to a previously published protocol [14]. This review indicates that there is a paucity of high-quality evidence to support any particular recommendations for reducing the occurrence of HIRRT in critically ill patients. The most current Kidney Disease Improving Global Outcomes (KDIGO) AKI guidelines do not have recommendations in this regard [56]. French guidelines, based on expert opinion [61], suggest that for critically ill patients the use of higher dialysate sodium concentration, lower dialysate temperature, slow blood flow rates, and bicarbonate buffer be used for IHD. These recommendations accord with the findings of our systematic review. Nonetheless, such interventions (as well as novel ones) warrant more research given that the pathophysiology of HIRRT is particularly complex in critically ill patients [1]. Although a study did show that preload dependence prior to RRT initiation can predict HIRRT [62], there is also evidence that most HIRRT is unrelated to preload dependence [63]. As such, preload reduction from UF may often not be the primary driver of HIRRT in this population [44, 63]. Thus, the role of other potentially modifiable RRT-related factors in provoking HIRRT need to be better defined, with strategies developed and tested to mitigate them.

\section{Conclusion}

We identified only five RCTs and four observational studies that assessed RRT-related interventions aimed at reducing HIRRT among critically ill patients with AKI who received RRT. These studies were generally small, likely underpowered, and mostly of low quality. Overall, there is no definitive evidence to support the routine use of any particular RRT-related intervention to limit HIRRT in this population. However, from the data available, and consistent with some current guidelines [61], the use of higher dialysate sodium or sodium modeling, lower dialysate temperature, and slower blood flow rates for patients at risk of HIRRT should be considered in most cases. The lack of a consistent definition for HIRRT presents an impediment for further study. Establishing a uniform definition of HIRRT that is able to encompass drops in blood pressure as well as interventions taken in response to hemodynamic instability (e.g., fluid boluses, UF cessation) across different RRT modalities will be challenging. Nonetheless, doing so could help facilitate the design and execution of future trials testing interventions to prevent or mitigate HIRRT and its consequences.

\section{Additional file}

Additional file 1: Results According to RRT Modality. (PDF $131 \mathrm{~kb}$ )

\section{Abbreviations}

AKI: Acute kidney injury; CRRT: Continuous renal replacement therapy; CWH: Continuous venovenous hemofiltration; ESRD: End-stage renal disease; HIRRT: Hemodynamic instability related to renal replacement therapy; ICU: Intensive care unit; IDH: Intradialytic hypotension; IHD: Intermittent hemodialysis; KDIGO: Kidney Disease Improving Global Outcomes; MAP: Mean arterial pressure; NOS: Newcastle-Ottawa Scale; PPI: Peripheral perfusion index; RCT: Randomized controlled trial; RRT: Renal replacement therapy; SBP: Systolic blood pressure; SLED: Sustained low-efficiency dialysis; UF: Ultrafiltration

\section{Acknowledgements}

SMB is supported by a Canada Research Chair in Critical Care Nephrology.

Funding

Institutional resources only.

Availability of data and materials

All data generated and/or analyzed during this study are included in this published article.

\section{Authors' contributions}

Data collection, $A D, G M$, and EGC; study design, EGC, SH, LM, LS, and AD; data analysis, EGC, $A D$, and $S H$; manuscript preparation, EGC, AD, SH, and $L M$; review of the manuscript, $A D, G M, S H, L M, S A S, S M B, R W, C R, L S, C W$, and EGC. All authors read and approved the final manuscript.

Ethics approval and consent to participate

Not applicable.

\section{Consent for publication}

Not applicable.

\section{Competing interests}

$\mathrm{SMB}$ and RW have consulted and received speaker fees and grant support from Baxter Healthcare Corp. CR is an associate editor of Critical Care. The remaining authors declare that they have no competing interests.

\section{Publisher's Note}

Springer Nature remains neutral with regard to jurisdictional claims in published maps and institutional affiliations.

\footnotetext{
Author details

${ }^{1}$ Department of Medicine, University of Ottawa, Ottawa, ON, Canada. ${ }^{2}$ Division of Nephrology, Department of Medicine and Kidney Research Centre, Ottawa Hospital Research Institute, University of Ottawa, Ottawa, ON, Canada. ${ }^{3}$ Division of Critical Care, Department of Medicine, The Ottawa Hospital, Ottawa, ON, Canada. ${ }^{4}$ Centre for Transfusion Research, Clinical Epidemiology Program, Ottawa Hospital Research Institute, Ottawa, ON, Canada. ${ }^{5}$ Division of Nephrology, Queen's University, Kingston, ON, Canada. ${ }^{6}$ Department of Critical Care Medicine, Faculty of Medicine and Dentistry, University of Alberta, Edmonton, AB, Canada. ${ }^{7}$ Division of Nephrology, St. Michael's Hospital, Toronto, ON, Canada. ${ }^{8}$ International Renal Research Institute and Department of Nephrology, St. Bortolo Hospital, Vicenza, Italy. ${ }^{9}$ Health Sciences Library, University of Ottawa, Ottawa, ON, Canada. ${ }^{10}$ Division
} 
of Nephrology, McGill University, Montreal, Quebec, Canada. ${ }^{11}$ The Ottawa Hospital - Riverside Campus, 1967 Riverside Drive, Ottawa, ON K1H 7W9, Canada.

Received: 28 October 2017 Accepted: 24 January 2018 Published online: 22 February 2018

\section{References}

1. Sharma S, Waikar SS. Intradialytic hypotension in acute kidney injury requiring renal replacement therapy. Semin Dial. 2017;30:553-558.

2. Tonelli MAP, Andreou P, Beed S, Lundrigan P, Jindal K. Blood volume monitoring in intermittent hemodialysis for acute renal failure. Kidney Int. 2002:62:1075-80

3. Mehta R. Therapeutic alternatives to renal replacement for critically ill patients in acute renal failure. Semin Nephrol. 1994;14:64-82.

4. Tanguay TA, Jensen L, Johnston C. Predicting episodes of hypotension by continuous blood volume monitoring among critically ill patients in acute renal failure on intermittent hemodialysis. Dynamics. 2007;18(3):19-24.

5. Kitchlu A, Adhikari N, Burns KEA, Friedrich JO, Garg AX, Klein D, Richardson RM, Wald R. Outcomes of sustained low efficiency dialysis versus continuous renal replacement therapy in critically ill adults with acute kidney injury: a cohort study. BMC Nephrol. 2015;16:127.

6. Bagshaw SM, Darmon M, Ostermann M, Finkelstein FO, Wald R, Tolwani AJ, Goldstein SL, Gattas DJ, Uchino S, Hoste EA, et al. Current state of the art for renal replacement therapy in critically ill patients with acute kidney injury. Intensive Care Med. 2017:43(6):841-54.

7. Akhoundi A, Singh B, Vela M, Chaudhary S, Monaghan M, Wilson GA, Dillon JJ, Cartin-Ceba R, Lieske JC, Gajic O, et al. Incidence of adverse events during continuous renal replacement therapy. Blood Purif. 2015;39:333-9.

8. Uchino S, Bellomo R, Morimatsu H, Morgera S, Schetz M, Tan I, Bouman C, Macedo E, Gibney N, Tolwani A, et al. Continuous renal replacement therapy: a worldwide practice survey. The beginning and ending supportive therapy for the kidney (BEST Kidney) investigators. Intensive Care Med. 2007; 33:1563-70.

9. Silversides JAPR, Kuint R, Wald R, Hladunewich MA, Lapinsky SE, Adhikari NKJ. Fluid balance, intradialytic hypotension, and outcomes in critically ill patients undergoing renal replacement therapy: a cohort study. Crit Care. 2014;18:624.

10. Manns MSM, Teehan BP. Intradialytic renal haemodynamics - potential consequences for the management of the patient with acute renal failure. Nephrol Dial Transplant. 1997;12:870-2.

11. Cerda J, Liu KD, Cruz DN, Jaber BL, Koyner JL, Heung M, Okusa MD, Faube S. Nephrology AKIAGotASo. Promoting kidney function recovery in patients with AKI requiring RRT. Clin J Am Soc Nephrol. 2015;10(10):1859-67.

12. Forni LG, Darmon M, Ostermann M, Oudemans-van Straaten HM, Pettila V, Prowle JR, Schetz M, Joannidis M. Renal recovery after acute kidney injury. Intensive Care Med. 2017:43(6):855-66.

13. Palevsky PM, Baldwin I, Davenport A, Goldstein S, Paganini E. Renal replacement therapy and the kidney: minimizing the impact of renal replacement therapy on recovery of acute renal failure. Curr Opin Crit Care. 2005;11(6):548-54

14. Douvris A, Hiremath S, McIntyre L, Sikora L, Weber C, Clark EG. Interventions to prevent hemodynamic instability during renal replacement therapy for acute kidney injury: a systematic review protocol. Syst Rev. 2017;6(1):113

15. Wells GA SB, O'Connell D, Peterson J, Welch V, Losos M, Tugwell P: Newcastle-Ottawa Quality Assessment Scale Case Control Studies. Available at: http://www.ohri.ca/programs/clinical_epidemiology/nosgen.pdf. Accessed 30 Aug 2017.

16. Higgins JP, Altman DG, Gotzsche PC, Juni P, Moher D, Oxman AD, Savovic J, Schulz KF, Weeks L, Sterne JA, et al. The Cochrane Collaboration's tool for assessing risk of bias in randomised trials. BMJ. 2011;343:d5928.

17. Tian JH, Ma B, Yang K, Liu Y, Tan J, Liu TX. Bicarbonate- versus lactatebuffered solutions for acute continuous haemodiafiltration or haemofiltration. Cochrane Database Syst Rev. 2015:3:CD006819.

18. Eastwood GM, Peck L, Young H, Bailey M, Reade MC, Baldwin I, Bellomo R. Haemodynamic impact of a slower pump speed at start of continuous renal replacement therapy in critically ill adults with acute kidney injury: a prospective before-and-after study. Blood Purif. 2012;33(1-3):52-8.

19. du Cheyron D, Lucidarme O, Terzi N, Charbonneau P. Blood volume- and blood temperature-controlled hemodialysis in critically ill patients: a 6 month, case-matched, open-label study. Blood Purif. 2010;29(3):245-51.
20. du Cheyron D, Terzi N, Seguin A, Valette X, Prevost F, Ramakers M, Daubin C, Charbonneau P, Parienti JJ. Use of online blood volume and blood temperature monitoring during haemodialysis in critically ill patients with acute kidney injury: a single-centre randomized controlled trial. Nephrol Dial Transplant. 2013;28(2):430-7.

21. Lynch KE, Ghassemi F, Flythe JE, Feng M, Ghassemi M, Celi LA, Brunelli SM. Sodium modelling to reduce intradialytic hypotension during haemodialysis for acute kidney injury in the intensive care unit. Nephrology. 2016;21(10): 870-7.

22. Schortgen F, Soubrier N, Delclaux C, Thuong M, Girou E, Brun-Buisson C, Lemaire F, Brochard L. Hemodynamic tolerance of intermittent hemodialysis in critically ill patients: usefulness of practice guidelines. Am J Respir Crit Care Med. 2000;162(1):197-202

23. Lima EQ, Silva RG, Donadi EL, Fernandes AB, Zanon JR, Pinto KR, Burdmann EA. Prevention of intradialytic hypotension in patients with acute kidney injury submitted to sustained low-efficiency dialysis. Ren Fail. 2012;34(10): $1238-43$.

24. Albino BB, Balbi AL, Ponce D. Dialysis complications in AKI patients treated with extended daily dialysis: is the duration of therapy important? Biomed Res Int. 2014;2014:153626.

25. Robert R, Mehaud JE, Timricht N, Goudet V, Mimoz O, Debaene B. Benefits of an early cooling phase in continuous renal replacement therapy for ICU patients. Ann Intensive Care. 2012;2(1):40.

26. Paganini EP, Sandy D, Moreno L, Kozlowski L, Sakai K. The effect of sodium and ultrafiltration modelling on plasma volume changes and haemodynamic stability in intensive care patients receiving haemodialysis for acute renal failure: a prospective, stratified, randomized, cross-over study. Nephrol Dial Transplant. 1996;11(Suppl 8):32-7.

27. Dheenan S, Henrich WL. Preventing dialysis hypotension: a comparison of usual protective maneuvers. Kidney Int. 2001;59:1175-81.

28. Brummelhuis WJ, van Geest RJ, van Schelven LJ, Boer WH. Sodium profiling, but not cool dialysate, increases the absolute plasma refill rate during hemodialysis. ASAIO J. 2009;55(6):575-80.

29. Mendoza JM, Arramreddy R, Schiller B. Dialysate sodium: choosing the optimal hemodialysis bath. Am J Kidney Dis. 2015;66(4):710-20.

30. Song JH, Lee SW, Suh C-K, Kim M-J. Time-averaged concentration of dialysate sodium relates with sodium load and interdialytic weight gain during sodium-profiling hemodialysis. Am J Kidney Dis. 2002;40:291-301.

31. Song JH, Park GH, Lee SY, Lee SW, Kim M-J. Effect of sodium balance and the combination of ultrafiltration profile during sodium profiling hemodialysis on the maintenance of the quality of dialysis and sodium and fluid balances. J Am Soc Nephrol. 2005;16:237-46.

32. Kalantar-Zadeh K, Regidor DL, Kovesdy CP. Fluid retention is associated with cardiovascular mortality in patients undergoing long-term hemodialysis. Circulation. 2009:119:671-9.

33. Flythe JE, Curhan GC, Brunelli SM. Disentangling the ultrafiltration ratemortality association: the respective roles of session length and weight gain. Clin J Am Soc Nephrol. 2013;8:1151-61.

34. Basile C, Pisano A, Lisi P, Rossi L, Lomonte C, Bolignano D. High versus low dialysate sodium concentration in chronic haemodialysis patients: a systematic review of 23 studies. Nephrol Dial Transplant. 2016;31(4):548-63.

35. Silversides JA, Pinto R, Kuint R, Wald R, Hladunewich MA, Lapinsky SE, Adhikari NK. Fluid balance, intradialytic hypotension, and outcomes in critically ill patients undergoing renal replacement therapy: a cohort study. Crit Care. 2014;18(6):624

36. Heung M, Wolfgram DF, Kommareddi M, Hu Y, Song PX, Ojo AO. Fluid overload at initiation of renal replacement therapy is associated with lack of renal recovery in patients with acute kidney injury. Nephrol Dial Transplant. 2012;27(3):956-61.

37. Vaara ST, Korhonen AM, Kaukonen KM, Nisula S, Inkinen O, Hoppu S, Laurila $\mathrm{J}$, Mildh L, Reinikainen M, Lund V, et al. Fluid overload is associated with an increased risk for 90-day mortality in critically ill patients with renal replacement therapy: data from the prospective FINNAKI study. Crit Care. 2012;16(5):R197.

38. Zhang L, Chen Z, Diao Y, Yang Y, Fu P. Associations of fluid overload with mortality and kidney recovery in patients with acute kidney injury: a systematic review and meta-analysis. J Crit Care. 2015;30(4): 860. e867-13

39. Odudu A, Mclntyre CW. An update on intradialytic cardiac dysfunction. Semin Dial. 2016;29(6):435-41. 
40. Odudu A, Eldehni MT, McCann GP, Mclntyre CW. Randomized controlled trial of individualized dialysate cooling for cardiac protection in hemodialysis patients. Clin J Am Soc Nephrol. 2015;10:1408-17.

41. Selby NM, Mclntyre CW. A systematic review of the clinical effects of reducing dialysate fluid temperature. Nephrol Dial Transplant. 2006;21(7): 1883-98.

42. Selby NM, Burton JO, Chesterton LJ, McIntyre CW. Dialysis-induced regional left ventricular dysfunction is ameliorated by cooling the dialysate. Clin J Am Soc Nephrol. 2006;1(6):1216-25.

43. Mustafa RA, Bdair F, Akl EA, Garg AX, Thiessen-Philbrook H, Salameh H, Kisra S, Nesrallah G, Al-Jaishi A, Patel P, et al. Effect of lowering the dialysate temperature in chronic hemodialysis: a systematic review and meta-analysis. Clin J Am Soc Nephrol. 2016;11:442-57.

44. Mahmoud H, Forni LG, Mclntyre CW, Selby NM. Myocardial stunning occurs during intermittent haemodialysis for acute kidney injury. Intensive Care Med. 2017;43(6):942-4

45. Marat S, Salerno F, Ball I, Ellis C, Mclntyre C. Continuous renal replacement therapy is associated with acute myocardial injury in critically ill patients. Intensiv Care Med Exp. 2017;5(Suppl 2):0847.

46. Rokyta R, Matejovic M, Krouzecky A, Opatrny K Jr, Ruzicka J, Novak I. Effects of continuous venovenous haemofiltration-induced cooling on global haemodynamics, splanchnic oxygen and energy balance in critically ill patients. Nephrol Dial Transplant. 2004;19:623-30.

47. Yagi N, Leblanc M, Sakai K, Wright EJ, Paganini EP. Cooling effect of continuous renal replacement therapy in critically ill patients. Am J Kidney Dis. 1998;32(6):1023-30.

48. Curley FJ. Hypothermia: a critical problem in the intensive care unit. J Intensive Care Med. 1995;10:1-2.

49. Manthous CA, Hall JB, Olsen D, Singh M, CHatila W, Pohlman A, Kushner R, Schmidt GA, Wood LD. Effect of cooling on oxygen consumption in febrile critically ill patients. Am J Respir Crit Care Med. 1995;151(1):10-4.

50. Ronco C, Brendolan A, Milan M. Impact of biofeedback-induced cardiovascular stability on hemodialysis tolerance and efficiency. Kidney Int. 2000;58:800-8.

51. Steuer RR, Germain MJ, Leypoldt JK, Cheung AK. Reducing symptoms during hemodialysis by continuously monitoring the hematocrit. Am J Kidney Dis. 1996;27:525-32.

52. Shulman T, Heidenheim AP, Kianfar C. Preserving central blood volume: changes in body fluid compartments during hemodialysis. ASAIO J. 2001;47: 615-8.

53. Klijn E, Groeneveld ABJ, Van Genderen ME, Betjes M, Bakker J, Van Bomme J. Peripheral perfusion index predicts hypotension during fluid withdrawal by continuous veno-venous hemofiltration in critically ill patients. Blood Purif. 2015;40(1):92-8.

54. Kim IB, Fealy N, Baldwin I, Bellomo R. Circuit start during continuous renal replacement therapy in vasopressor-dependent patients: the impact of a slow blood flow protocol. Blood Purif. 2011;32:1-6.

55. Reilly RF. Attending rounds: a patient with intradialytic hypotension. Clin J Am Soc Nephrol. 2014;9:798-803.

56. Kidney Disease Improving Global Outcomes (KDIGO) Acute Kidney Injury Work Group. KDIGO clinical practice guidelines for acute kidney injury. Kidney inter Suppl. 2012;2:1-138.

57. Bagshaw SM, Berthiaume LR, Delaney A, Bellomo R. Continuous versus intermittent renal replacement therapy for critically ill patients with acute kidney injury: a meta-analysis. Crit Care Med. 2008;36(2):610-7.

58. Kovacs B, Sullivan KJ, Hiremath S, Patel RV. Effect of sustained low efficient dialysis versus continuous renal replacement therapy on renal recovery after acute kidney injury in the intensive care unit: a systematic review and metaanalysis. Nephrology. 2017;22(5):343-53.

59. Schneider AG, Bellomo R, Bagshaw SM, Glassford NJ, Lo S, Jun M, Cass A, Gallagher M. Choice of renal replacement therapy modality and dialysis dependence after acute kidney injury: a systematic review and metaanalysis. Intensive Care Med. 2013;39(6):987-97.

60. Zhang L, Yang J, Eastwood GM, Zhu G, Tanaka A, Bellomo R. Extended daily dialysis versus continuous renal replacement therapy for acute kidney injury: a meta-analysis. Am J Kidney Dis. 2015;66(2):322-30.

61. Vinsonneau C, Allain-Launay E, Blayau C, Darmon M, Ducheyron D, Gaillot T, Honore PM, Javouhey E, Krummel T, Lahoche A, et al. Renal replacement therapy in adult and pediatric intensive care: recommendations by an expert panel from the French Intensive Care Society (SRLF) with the French Society of Anesthesia Intensive Care (SFAR) French Group for Pediatric
Intensive Care Emergencies (GFRUP) the French Dialysis Society (SFD). Ann Intensive Care. 2015;5(1):58.

62. Monnet X, Cipriani F, Camous L, Sentenac P, Dres M, Krastinova E, Anguel N, Richard C, Teboul J-L. The passive leg raising test to guide fluid removal in critically ill patients. Ann Intensive Care. 2016;6:46.

63. Bitker L, Bayle F, Yonis H, Gobert F, Leray V, Taponnier R, Debord S, StoianCividjian A, Guerin C, Richard JC. Prevalence and risk factors of hypotension associated with preload-dependence during intermittent hemodialysis in critically ill patients. Crit Care. 2016;20:44.

\section{Submit your next manuscript to BioMed Central and we will help you at every step:}

- We accept pre-submission inquiries

- Our selector tool helps you to find the most relevant journal

- We provide round the clock customer support

- Convenient online submission

- Thorough peer review

- Inclusion in PubMed and all major indexing services

- Maximum visibility for your research

Submit your manuscript at www.biomedcentral.com/submit
Biomed Central 